\title{
GROUP ACTIONS ON POINCARÉ DUALITY SPACES
}

\author{
BY THEODORE CHANG AND TOR SKJELBRED \\ Communicated by S. S. Chern, April 21, 1972
}

Let $G=Z_{p}$ for $p$ prime and $K=Z_{p}$, or let $G=S^{1}$ and $K=Q$, and let $G$ act on the compact space $X$. In this paper, we outline two proofs of the following:

Theorem. Suppose the compact G-space $X$ is a Poincare duality space over $K$ of formal dimension $n$. Then each connected component of the fixed point set is a Poincaré duality space over $K$, and, if $G \neq Z_{2}$, has formal dimension congruent to $n$ mod 2 .

This solves affirmatively the conjecture of Su given in [5].

Let $E_{G} \rightarrow B_{G}$ be the universal bundle for $G$ and let $X_{G}$ be the balanced product $\left(X \times E_{G}\right) / G$. The basic tools for both proofs are the fibre space $X \rightarrow X_{G} \rightarrow B_{G}$ and the localization theorem of Borel ([1], [4]). In the case $X$ is totally nonhomologous to zero in $X_{G}$, Bredon has proven the $\mathrm{Su}$ conjecture [2]. However, this condition can be replaced by the two lemmas below, and this constitutes our algebraic proof. The second proof involves applying the localization theorem to a Thom space.

We wish to thank our advisor Wu-yi Hsiang for his suggestions and encouragement.

1. Algebraic proof. When $G=S^{1}$ or $Z_{2}, H^{*}\left(B_{G}\right)=K[t]$ where $t$ is of degree two in the $S^{1}$ case and of degree one in the $Z_{2}$ case. If $G=Z_{p}$ for $p$ odd, then $H^{*}\left(B_{G}\right)=K[t, s] / s^{2}=0$ where $s$ has degree one and $t$ degree two. We consider the cohomology spectral sequence of the fibre space $X \rightarrow X_{G} \rightarrow B_{G}$.

Lemma $1 .\left(1_{r}\right) E_{r}$ is generated over $K[t]$ by $E_{r}^{0, *}$ and $E_{r}^{1, *}$ for $G \neq Z_{2}$ or $S^{1}$, and by $E_{r}^{0, *}$ for $G=Z_{2}$ or $S^{1}$.

$\left(2_{r}\right)$ If $j \geqq r-1$, cup product with $t$ gives an isomorphism of $E_{r}^{j, k}$ into $E_{r}^{j+2, k}$ for $G \neq Z_{2}$ and of $E_{r}^{j, k}$ into $E_{r}^{j+1, k}$ for $G=Z_{2}(r \geqq 2)$.

Lemma 2. Suppose there is a fixed point. Then the fundamental class $U$ of $H^{n}(X)$ survives in $E_{\infty}^{0, n}$ and if $u \in E_{\infty}^{0, *}$ is nontorsion with respect to $H^{*}\left(B_{G}\right)$, there exists $a v \in E_{\infty}^{0, *}$ such that $u v=U$ (cup product).

Lemma 1 is proven by induction. $1_{2}$ and $2_{2}$ are true for $G=S^{1}$ since $E_{2}=H^{*}\left(B_{G}\right) \otimes H^{*}(X)$, and for $G=Z_{p}$ by known results of homological

AMS. 1970 subject classifications. Primary 55C35, 57B10; Secondary 55F20.

Copyright (C) American Mathematical Society 1972 
algebra (see [3]). The induction step is then shown by straightforward degree arguments.

Lemma 2 is proven by restriction to a $N$-dimensional orientable submanifold $B \subset B_{G}$ for large $N$. Then since $H^{n}\left(X, Z_{p}\right)=Z_{p}$ and $Z_{p}$ has no nontrivial action on $Z_{p}$, the local coefficients are trivial in the top dimension. Thus by piecing together over neighborhoods on which $\left.X_{G}\right|_{B}$ is trivial, it is easy to show that $\left.X_{G}\right|_{B}$ satisfies Poincare duality with a cohomology fundamental class $[B] U$ where $\left.X_{G}\right|_{B}$ is the portion of $X_{G}$ over $B$ and $[B]$ is the fundamental class of $B$. Using the fact that the inclusion $\left.X_{G}\right|_{B} \rightarrow X_{G}$ induces an isomorphism on $E_{2}^{j, k}$ for $j \leqq N$ implies it induces an injection on $E_{\infty}^{j, k}$ for $j \leqq N$, and choosing $N$ large enough so it induces an isomorphism on $E_{\infty}^{0, *}$, the lemma follows by finding a class in $H^{*}\left(\left.X_{G}\right|_{B}\right)$ dual to $[B] u$.

With these two lemmas the proof of Bredon is valid without change.

2. Geometric proof. We shall assume that

(i) $X$ can be embedded in Euclidean space as a neighborhood retract.

(ii) $X$ has a finite number of orbit types.

Property (i) is inherited by the fixed point set, as follows from (ii) and the equivariant embedding theorem. Because of (i) $X$ is a Poincaré duality space over $K$ of formal dimension $n$ if and only if for any embedding $X \subset S^{n+r}=S$ there is an isomorphism

$$
x \mapsto x \cup U: H^{*}(X) \rightarrow H^{*}(S, S-X)
$$

for some $U \in H^{r}(S, S-X)$.

Choose a $G$-equivariant embedding, and use $K$ as the coefficient field. Then $H^{r}(S, S-X)=H_{G}^{r}(S, S-X)$ and we consider $U$ as an element of both groups, where we define for any $G$-pair $\left(A, A^{\prime}\right), H_{G}^{*}\left(A, A^{\prime}\right)$ $=H^{*}\left(A_{G}, A_{G}^{\prime}\right)$. By induction on the cells in $B_{G}$, we see that there is an isomorphism

$$
\cup U: H_{G}^{*}(X) \rightarrow H_{G}^{*}(S, S-X) .
$$

Let $\Sigma$ be the fixed sphere in $S$ and $F=X \cap \Sigma$ the fixed set in $X$. Then the following diagram is commutative:

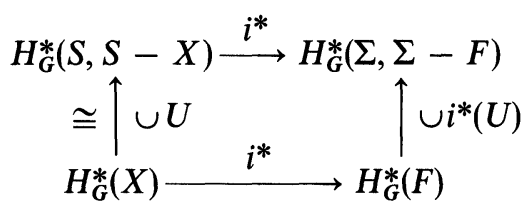

After localizing, the maps $i^{*}$ and hence all the maps in the diagram are isomorphisms. Localizing here means tensoring over $K[t] \subset H^{*}\left(B_{G}\right)$ with $K\left[t, t^{-1}\right]$. The map on the right splits according to the connected 
components of $F$, so we may assume $F$ is connected. Then

$$
i^{*}(U)=t^{a} u_{0}+t^{a-1} u_{1}+\ldots+u_{a}+s v
$$

where $u_{i} \in H^{*}(\Sigma, \Sigma-F), u_{0} \neq 0$, and $s=0$ if $G=Z_{2}$ or $S^{1}$. Hence

$$
\cup u_{0}: H^{*}(F) \rightarrow H^{*}(\Sigma, \Sigma-F)
$$

is an isomorphism, so $F$ is a Poincaré duality space over $K$.

\section{REFERENCES}

1. A. Borel, Seminar on transformation groups, Ann. of Math. Studies, no. 46, Princeton Univ. Press, Princeton, N.J., 1960. MR 22 \# 7129.

2. G. Bredon, The cohomology ring structure of a fixed point set, Ann. of Math. (2) 80 (1964), 524-537. MR 32 \# 1698.

3. H. Cartan and S. Eilenberg, Homological algebra, Princeton Univ. Press, Princeton, N.J., 1956. MR 17, 1040.

4. W. Y. Hsiang, On some fundamental theorems in cohomology theory of topological transformation groups, Taita (Nat. Taiwan Univ.) J. Math. 2 (1970), 61-87.

5. J. C. Su, Periodic transformations on the product of two spheres, Trans. Amer. Math. Soc. 112 (1964), 369-380. MR 29 \#612.

Department of Mathematics, University of California, Berkeley, California 94720 (Current address of Tor Skjelbred)

Current address (Theodore Chang): Department of Mathematics, University of Kansas, Lawrence, Kansas 66044 